\title{
Complete amenorrhea after treatment of resistant menorrhagia using NovaSure ablation
}

\author{
Ibrahim A. Abdelazim¹,2, Mohannad AbuFaza', Shikanova Svetlana³, Gulmira Zhurabekova ${ }^{4}$ \\ 'Department of Obstetrics and Gynecology, Ahmadi Hospital, Kuwait Oil Company (KOC), Kuwait \\ ${ }^{2}$ Department of Obstetrics and Gynecology, Ain Shams University, Cairo, Egypt \\ ${ }^{3}$ Department of Obstetrics and Gynecology, Marat Ospanov, West Kazakhstan State Medical University, Aktobe, Kazakhstan (WKSMU) \\ ${ }^{4}$ Department of Normal and Topographical Anatomy, Marat Ospanov, West Kazakhstan State Medical University, Aktobe, \\ Kazakhstan (WKSMU)
}

\begin{abstract}
Introduction: Heavy menstrual bleeding $(\mathrm{HMB})$ or menorrhagia is the most common form of dysfunctional uterine bleeding (DUB). In spite of medical treatment for DUB, many women will eventually require a hysterectomy, which is an invasive treatment option. NovaSure ablation offers a same-day non-invasive alternative to hysterectomy and hysteroscopic ablation.

Case description: A 38-year-old woman presented with HMB in 2015. The attacks of HMB interrupted her life-style, and she refused to continue with medical treatment, which failed to resolve her symptoms. The patient was admitted to the hospital four times in 2015, due to anemia caused by the DUB. Medroxyprogesterone acetate and oral contraceptive pills failed to control the patient's bleeding episodes. Hysteroscopic examination of the uterine cavity showed a normal cavity, and the endometrial biopsy showed proliferative endometrium. She was counseled about NovaSure ablation as the last treatment option before hysterectomy. The NovaSure ablation procedure took $90 \mathrm{~s}$, and the patient was discharged from the hospital $6 \mathrm{~h}$ after the procedure. At follow-up, the patient is completely amenorrheic, and she is satisfied with the NovaSure ablation results.

Conclusions: This case report demonstrates that NovaSure endometrial ablation is a safe, effective, non-invasive alternative to hysteroscopic endometrial ablation for treatment of DUB.

Key words: amenorrhea, NovaSure, ablation, menorrhagia.
\end{abstract}

Ibrahim A. Abdelazim, Department of Obstetrics and Gynecology, Ain Shams University, and Ahmadi Hospital, Kuwait Oil Company (KOC), P.O. Box 9758, Ahmadi 61008, Kuwait, phone: +965 66551300, e-mail: dr.ibrahimanwar@gmail.com 


\section{Introduction}

Heavy menstrual bleeding (HMB) or menorrhagia is the most common form of dysfunctional uterine bleeding (DUB). Dysfunctional uterine bleeding is diagnosed after exclusion of any pelvic pathology and/or medical causes of bleeding [1].

Heavy menstrual bleeding or menorrhagia is the most common form of DUB, affecting 15-20\% of women during their reproductive period. The DUB has significant adverse effects on the quality of life [2].

In spite of medical treatment for DUB, many women will eventually require a hysterectomy, as an invasive treatment option [3].

Hysteroscopic endometrial ablation, and recently NovaSure endometrial ablation, were developed as non-invasive treatment options for DUB after failed medical treatment [4].

Compared to hysteroscopic techniques which require proper visualization and a skilled surgeon, the NovaSure ablation technique does not require proper visualization, is less dependent on the surgeon's skills, and eliminates the risk of fluid overload [5].

We present a case report of a relatively young woman, who had suffered from DUB for many years, and had frequent hospital admissions and absences from work due to this condition. This woman was successfully treated with NovaSure ablation, and she has remained symptom free for one and half years after NovaSure ablation, and has been able to resume her usual daily activities and life-style practices.

\section{Case description}

A 38-year-old woman with a history of two previous cesarean sections presented with HMB lasting for 1 year. She complained that the bleeding episodes interrupted her work and life-style. She refused to continue medical treatment options, which had failed to resolve her symptoms. The patient had normal thyroid function, normal liver functions, and normal coagulation and bleeding profiles. Her post-menstrual transvaginal scan to exclude intrauterine lesions (fibroids or polyps) showed a normal uterus $(8-10 \mathrm{~cm}$

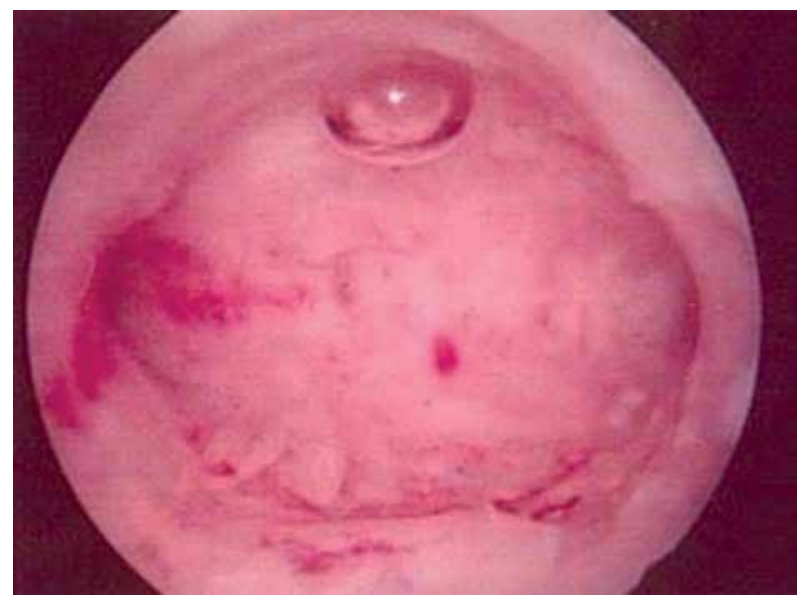

Figure 1. Hysteroscopic examination of the uterus prior to ablation in length), without any intrauterine lesions, and normal ovaries. The patient had a normal Pap smear, normal hysteroscopic evaluation of the uterine cavity, and the endometrial biopsy taken 3 months before showed disordered proliferative endometrium. The patient was admitted to the hospital four times over the course of a year due to anemia caused by attacks of HMB.

During her first admission, her hemoglobin was $9 \mathrm{~g} / \mathrm{dl}$. She received medical treatment for menorrhagia in the form of medroxyprogesterone acetate $10 \mathrm{mg}$ daily for 10 days each month for 3 months with tranexamic acid (anti-fibrinolysis) 1-1.5 g 3 times/day, and parenteral iron was used to correct the patient's anemia [6].

At her second admission, her hemoglobin was $9.5 \mathrm{~g} / \mathrm{dl}$. She received medical treatment in the form of oral contraceptive pills twice daily for 7 days, followed by 1 pill/day after reduction of the menstrual flow for the rest of the cycle for 3 months with tranexamic acid, and parenteral iron was used to correct the patient's anemia [6].

During the third admission, the patient received blood transfusion (hemoglobin was $7 \mathrm{~g} / \mathrm{dl}$ ). She was counseled about diagnostic hysteroscopy under general anesthesia for evaluation of the uterine cavity with an endometrial biopsy, and insertion of a levonorgestrel intra-uterine device (IUD) [6].

The hysteroscopic evaluation showed a normal uterine size, without any gross intrauterine lesions such as fibroids or polyps, and the histological examination of the endometrial biopsy taken during hysteroscopy showed disordered proliferative endometrium. In addition, the patient was counseled for the possibility of NovaSure endometrial ablation if the levonorgestrel IUD failed to control the bleeding episodes, because she had a normal sized uterine cavity, without intrauterine lesions, and disordered proliferative endometrium (Figure 1).

The studied woman was admitted again two months after insertion of the levonorgestrel IUD due to a severe attack of menorrhagia with expulsion of the levonorgestrel IUD. During the last admission, she received parenteral iron for correction of anemia. She decided to proceed with NovaSure ablation. Informed consent was obtained including the risks for future pregnancies following NovaSure ablation, the risks of the ablation procedure (thermal injury, perforation, infection), and post-operative side effects (cramps, discharge, and spotting).

In addition, the patient was informed about the benefits of the NovaSure endometrial ablation, such as the advantage of a non-hormonal, non-invasive procedure to control the bleeding attacks, and was informed about hysterectomy as the last treatment option if NovaSure ablation failed.

NovaSure endometrial ablation was performed immediately after correction of the patient's general condition under general anesthesia, and after endometrial curettage, because a better outcome is obtained if endometrial ablation is done with endometrial thickness $<4 \mathrm{~mm}$ [7].

Hysteroscopic examination of the uterine cavity after NovaSure endometrial ablation is not mandatory. It was performed in this case in order to counsel the patient about 
the expected results after the ablation procedure. The hysteroscopic examination performed after NovaSure ablation showed complete ablation of the endometrium (Figures 2, 3), and the patient was counseled that she should expect complete amenorrhea after the procedure. The ablation procedure took $90 \mathrm{~s}$ without any intra-operative or post-operative complications. The patient was discharged from the hospital $6 \mathrm{~h}$ after the procedure on non-steroidal anti-inflammatory drugs (as analgesics).

At follow-up, the patient was completely amenorrheic, and she was able to resume her active life style. The patient is completely satisfied with the results of the ablation procedure, and her symptoms have completely resolved.

\section{Discussion}

NovaSure is a second generation non-invasive endometrial ablation procedure that is completed in less than $3 \mathrm{~min}$ (100-120 s), and was approved by the FDA in 2001 [8].

NovaSure endometrial ablation is indicated for women with DUB after exclusion of endocrine or coagulation disorders, gross uterine cavity pathology (uterine polyps or fibroids), and active genital or pelvic infection [8].

Second generation ablation techniques are quick, safe procedures, that do not require fluid media, and are less dependent on the surgeon's skills [9].

Prior systematic reviews concluded that the second generation ablation techniques were less time consuming compared to the first generation techniques, and can be performed under local anesthesia with similar success $[3,4]$.

Gallinat concluded that long-term clinical results demonstrate that the NovaSure system was a safe and effective method for treatment of DUB in 107 women studied, with a high success rate and low re-treatment rates [9].

Gallinat and Nugen reported that NovaSure is a safe, effective treatment option in DUB after failed medical treatment. In addition, the authors reported $98 \%$ successful reduction in the bleeding attacks with a $58.6 \%$ amenorrhea rate at 12 -month follow-up in 107 women with menorrhagia studied [10].

Kalkat and Cartmill studied 50 women with menorrhagia unresponsive to medical management, who were treated with endometrial ablation under local anesthesia. The authors reported $94 \%(47 / 50)$ improvement in the menstrual bleeding, with $86 \%(43 / 50)$ and $94 \%(47 / 50)$ satisfaction rates after 4 and 6 months of the NovaSure ablation, respectively [11].

In addition, the NovaSure ablation technique was evaluated by Thiel et al., in 87 women with uterine length $>10 \mathrm{~cm}$ compared to 101 women with uterine length $\leq 10 \mathrm{~cm}$ (101 women). The authors concluded that the NovaSure endometrial ablation was associated with successful outcomes in women with uterine length $>10 \mathrm{~cm}$, without any serious procedure-related adverse events [12].

Eighty-one women with HMB were randomly assigned by Clark et al. to bipolar radiofrequency endometrial ablation or thermal balloon ablation in an office setting. Clark et al. reported that the rate of amenorrhea was significant-

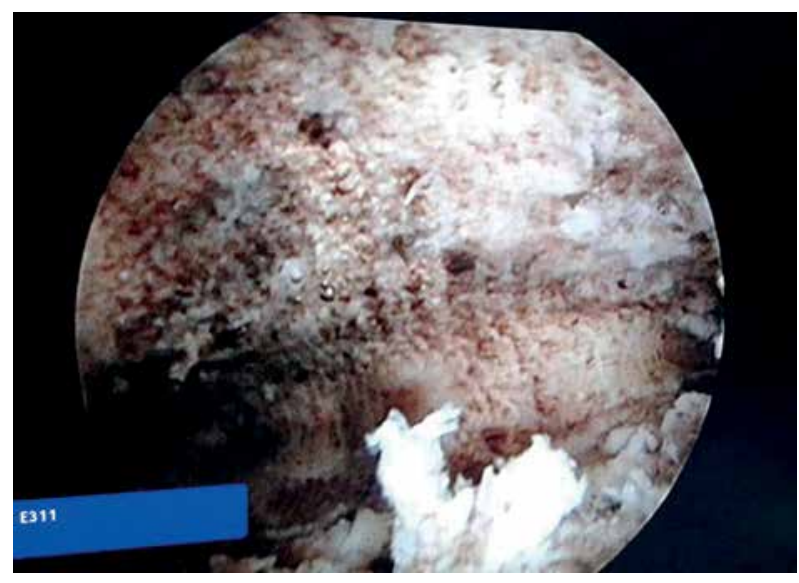

Figure 2. Hysteroscopic examination of the uterus after NovaSure ablation (far view)

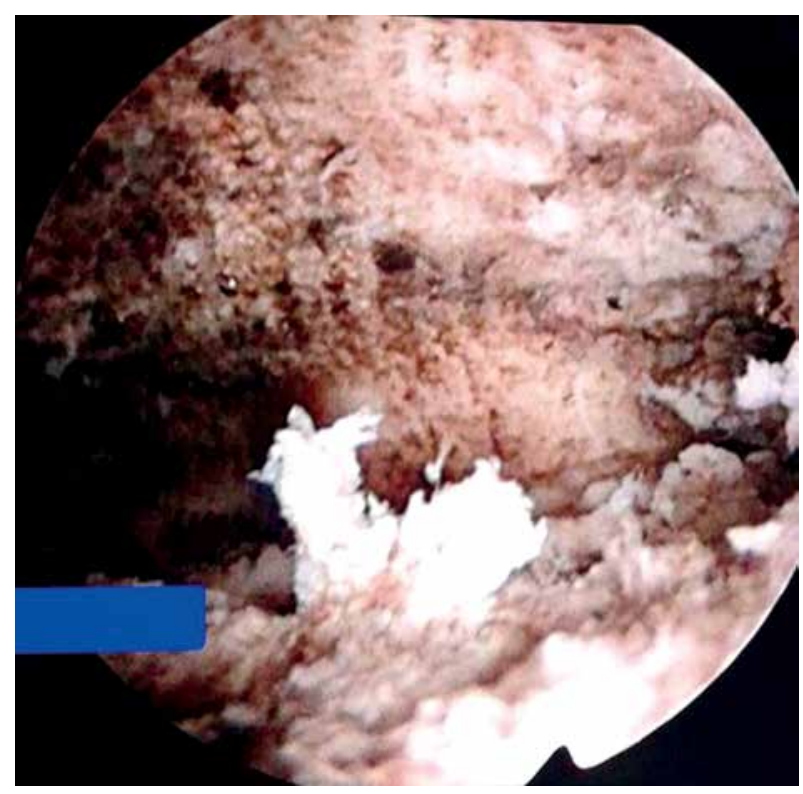

Figure 3. Hysteroscopic examination of the uterus after NovaSure ablation (near view)

ly higher in NovaSure-treated women (56\%) compared to ThermaChoice III-treated women (23\%) after 12-month follow-up [13].

In addition, Abbott et al. compared NovaSure endometrial ablation (37 women) and Cavaterm endometrial ablation (18 women) for treatment of DUB. Abbott et al. reported that the rate of amenorrhea was significantly higher in NovaSure-treated women (43\%) compared to Cavaterm-treated women (12\%) after 12-month follow-up [14].

NovaSure is a safe, non-invasive procedure, with minimal post-operative adverse events including pelvic cramps, nausea and/or vomiting, hematometra, and urinary tract infection. Endometritis after NovaSure ablation is uncommon, and is reported in $0.6-5 \%$ of cases [14].

In this case report, the patient was admitted to the hospital four times over a one-year period due to anemia from the bleeding episodes. The anemia was corrected by parenteral iron three times, and blood transfusion once. The medical treatment (medroxyprogesterone acetate, oral con- 
traceptive pills), and levonorgestrel IUD failed to control her bleeding episodes.

NovaSure endometrial ablation was performed for the patient, followed by hysteroscopic examination of the uterine cavity after NovaSure ablation (which is not mandatory). The hysteroscopic examination performed after NovaSure ablation showed complete ablation of the endometrium, and the patient was counseled to anticipate complete amenorrhea after the procedure.

At follow-up, the patient is completely amenorrheic and she has resumed her work and daily activity. She is completely satisfied, after 1 year of suffering from frequent hospital admissions, and absence from work, because of the heavy bleeding attacks.

Post-procedure patient counseling based on the hysteroscopic examination after NovaSure ablation, and the successful outcome after frequent hospital admissions, are the main reasons behind the presentation of the studied case as a case report.

In conclusion, NovaSure is an FDA-approved endometrial ablation technique for treatment of DUB after failed medical treatment and levonorgestrel IUD. NovaSure endometrial ablation is a safe, effective, noninvasive alternative to hysteroscopic endometrial ablation. Compared to hysteroscopic ablation techniques, NovaSure ablation does not require proper visualization, is less dependent on the surgeon's skills, and eliminates the risk of fluid overload.

\section{Acknowledgments}

The authors are grateful to the patient presented in this study for allowing her case to be presented.

\section{Conflict of interest}

The authors declare no conflict of interest.

\section{References}

1. Abdelazim IA, Abu-Faza M, Hamdan SB. Outcome and hemodynamic alternation in uterine artery following hysteroscopic roller-ball endometrial ablation. J Gynecol Res 2015; 1: 105.

2. Abdelazim IA, Belal MM. Outcome and uterine arteries Doppler velocimetry after thermal balloon endometrial ablation in menorrhagia. Evid Based Women Health J 2013; 3: 35-8.

3. Lethaby A, Hickey M, Garry R. Endometrial destruction techniques for heavy menstrual bleeding. Cochrane Database Syst Rev 2009; 4: CD001501.

4. Garside R, Stein K, Wyatt K, Round A, Price A. The effectiveness and cost-effectiveness of microwave and thermal balloon endometrial ablation for heavy menstrual bleeding: a systematic review and economic modelling. Health Technol Assess 2004; 8: 1-155.

5. Abbott JS, Garry R. The surgical management of menorrhagia. Hum Reprod Update 2002; 8: 68-78.

6. Wellington $\mathrm{K}$, Wagstaff AJ. Tranexamic acid: a review of its use in the management of menorrhagia. Drugs 2003; 63: 1417-33.

7. Practice Committee of American Society for Reproductive Medicine. Indications and options for endometrial ablation. Fertil Steril 2008; 90 (5 Suppl): S236-40.
8. Gimpelson RJ. Ten-year literature review of global endometrial ablation with the NovaSure ${ }^{\circledR}$ device. Int J Womens Health 2014; 6: 269-80.

9. Gallinat A. NovaSure impedance-controlled system for endometrial ablation: three-year follow-up on 107 patients. Am J Obstet Gynecol 2004; 191: 1585-9.

10. Gallinat A, Nugent W. NovaSure impedance-controlled system for endometrial ablation. J Am Assoc Gynecol Laparosc 2002; 9: 283-9.

11. Kalkat RK, Cartmill RS. NovaSure endometrial ablation under local anaesthesia in an outpatient setting: An observational study. J Obstet Gynecol 2011; 31: 152-5.

12. Thiel JA, Briggs MM, Pohlman S, Rattray D. Evaluation of the NovaSure endometrial ablation procedure in women with uterine cavity length over $10 \mathrm{~cm}$. J Obstet Gynaecol Can 2014; 36: 491-4.

13. Clark TJ, Samuels N, Malick S, Middleton L, Daniels J, Gupta J. Bipolar radiofrequency compared with thermal balloon endometrial ablation in the office: a randomized controlled trial. Obstet Gynecol 2011; 117: 109-11.

14. Abbott J, Hawe J, Hunter D, Garry R. A double blind randomized trial comparing the Cavaterm and the NovaSure endometrial ablation systems for the treatment of dysfunctional uterine bleeding. Fertil Steril 2003; 80: 203-8. 